\title{
On the Formation of Jets
}

\author{
Annalisa Celotti ${ }^{1}$ and Roger D. Blandford ${ }^{2}$ \\ 1 SISSA, via Beirut 2-4, 1-34014 Trieste, Italy \\ 2 Caltech, Pasadena, CA 91125, USA
}

\begin{abstract}
The phenomenology of jets associated with a variety of black hole systems is summarized, emphasizing the constraints imposed on their origin. Models of jet formation are reviewed, focusing in particular on recent ideas concerning MHD models. Finally, the potential for advancing our understanding of jets both through future observations - especially forthcoming X-ray missions - and for elucidating some crucial theoretical questions is highlighted.
\end{abstract}

\section{What We (Probably) See}

Jets - in the broad qualitative meaning of collimated/elongated structures of outflowing plasma - are observed in a variety of systems. Other contributions to these proceedings focus on Galactic sources and thus what follows is biased toward extragalactic jets. For exhaustive reviews see e.g. [1], [2], [3].

\subsection{Active Galactic Nuclei}

Extended double radio emitting structures (lobes) were observed and associated with galaxies and quasars more than 40 years ago, and eventually recognized to be physically connected, through jets supplying them with energy and momentum, to the activity taking place in the nucleus in (about $10 \%$ of) active galaxies [4]. Analysis of the extended structures provided us with an estimate of the energy supplied by the nuclear engine.

A step toward the understanding of the (magneto)-hydrodynamics of jets on large $(\operatorname{arcsec})$ scales and their interaction with the environment was the recognition that differences in radio morphologies correspond to differences in power. Edge darkened, low power or type I sources, usually show two jets plausibly at most transonic, while edge brightened, powerful type II sources are mostly one-sided and appear to be supersonic and mildly relativistic.

The rather poor information on large scale jets in other spectral bands has been significantly filled in by HST, which has so far detected more than a dozen jets, and X-ray images from Chandra are just starting to be obtained (PKS 0637-752 and Cen A so far). These observations, which show rather similar radio and optical morphologies, reveal: a) that particles are accelerated to energies $\sim 10^{8} m_{e} c^{2}[5]$ and b) a constancy of the radio-optical spectra along jets, suggesting that the acceleration of synchrotron emitting particles occurs all along the jet, although it is not clear whether this is due to many (relativistic) shocks or wave modes. 
However it has been the study of the predicted motion of features in the inner jets at relativistic speed - relative to the flat spectrum compact source - which has provided major clues and constraints on the jet formation mechanism, in terms of the (bulk) acceleration and collimation required. The strongest pieces of evidence include the detection of components apparently moving at superluminal speeds $\sim$ few-10 $\mathrm{ch}^{-1}$, brightness temperatures exceeding $10^{12} \mathrm{~K}$, and one-sided jets. Often extragalactic jets are aligned over many decades in scale, are collimated within a few degrees and reach bulk Lorentz factors $\sim 10$.

When the emission from the highly relativistic (non-thermal) plasma is beamed in the direction of the observer, it dominates the isotropic line and continuum emission associated with the accreting gas and the stars. This is observed in blazars. The implied anisotropy in the emission has led to identify the misaligned counterparts of blazars with radio galaxies and quasars, providing some degree of unification among jetted sources of both types I and II. Another source of anisotropy, due to a putative obscuring torus, is invoked to account for the lack of broad emission lines in the spectra of powerful radio galaxies (and type 2 Seyferts).

A strong stimulus to this field has come from the observations of $\gamma$-ray emission in a significant number of blazars. Blazar jets are commonly observed at $\mathrm{GeV}$ energies and, when located within an intergalactic absorption length, also at $\mathrm{TeV}$ energies. (Note that $\mathrm{TeV}$ emission has been observed to vary on 15 min timescales [6], suggesting that these high energy $\gamma$-rays originate within $\sim 10^{2-3} \mathrm{~m}$.) These observations allow us to observe most of the energy that is radiatively dissipated (of which the radio emission contributes a negligible amount). Furthermore, constraints derived from the implied opacity for $\gamma-$ rays to pair production, locate the emitting region at $\sim 10^{2-4} \mathrm{~m}$ and thus limit the possible radiative processes involved.

Jet structures have been also increasingly found in radio-quiet AGN. Although these are not radio silent, they appear to be a separate class from the radio-loud AGN. These are less powerful and less collimated, with plasma moving at sub or at most mildly relativistic velocities (up to $\sim 0.1 c$ ). They are observed as radio outflows and indirectly through Broad Absorption Lines (BAL) in the optical-UV spectra in about $10 \%$ of the powerful objects, possibly those observed at low latitudes through equatorial outflows.

\subsection{X-Ray Binaries}

In the last decade, evidence has also accumulated for jets being commonly associated with Galactic X-ray binary systems, possibly as many as $20 \%$ of them [7], and among these, a few transient objects intriguingly showing apparent superluminal motion. A peculiar case - although other neutron star binaries might present a similar behavior - is represented by the precessing jet associated with SS433, with the best -- although still unexplained - measured velocity of $0.26 \mathrm{c}$. See the contributions [7], [8], [9] on jets in Galactic sources. 


\subsection{And More...}

The zoology of jets should also include less powerful systems, in particular bipolar outflows associated with protostars (YSO), comprising different components, with gas in different ionizing states, and reaching velocities of a few hundred $\mathrm{km} \mathrm{s}^{-1}$. At the other extreme, jets or relativistic outflows seem to account best for the gamma-ray burst phenomenon and relativistic afterglow, with Lorentz factors believed to reach a few hundreds.

Although here we focus on jets associated with candidate black hole systems, the richness and diversity of conditions and environments in which jets are observed on one side reveals that jets are common features, relatively easy structures to form, but on the other cautions against the temptation of following the (morphological) similarity to understand their origin as rather different detailed mechanisms are likely to be at work in different systems.

\section{What We Dream}

What can we infer from the wealth of observations on why jets form, how they are energized, accelerated, collimated and confined, and what is the gas flow around the black hole?

The main piece of evidence which emerges from observing jets in different objects is the invariable association with an accretion disc, although possibly reflecting different accretion regimes. While not all disk systems appear to produce powerful quasi-stationary jets, weaker jets/outflows might always be present at some level, as a necessary condition for accretion to take place, by extracting angular momentum from the inflow (indeed a large scale outflow rather than a powerful well collimated jet on small scales might dominate this process). If so, this would also indicate that the powerful flows are an extra ingredient. The other indication is that jets have speeds comparable with the escape velocity from their sources. (Speaking loosely, this is ultrarelativistic in the case of black holes.)

There are three proposed general mechanisms for jet formation.

i) Hydrodynamic acceleration: An adiabatic fluid propagating in an external medium with decreasing pressure, provides a relatively simple and direct way of achieving hydrodynamical self-collimation and acceleration, due to the requirement for the fluid to pass through the sonic point [10]. However, the gas that would be required to confine the most powerful extragalactic jets would radiate an $\mathrm{X}$-ray flux far larger than has been observed. This mechanism could be appropriate for low power jets.

ii) Radiative acceleration: An alternative possibility is to consider the intense radiation field as responsible for the acceleration. Two of the difficulties associated with this hypothesis are: a) many sources with powerful jets have luminosities well below the conventional Eddington limit - and consequently insufficient for acceleration, even when more efficient absorption processes 
are considered (e.g.[11]); b) the drag caused by the radiation severely limits the attainable velocity. An independent confining mechanism would, in any case, be required if the jet is accelerated by a thin disk. Alternatively, while a funnel, which might form in the central part of a flow accreting onto a black hole when the fluid has enough (radiation or ion) pressure, can provide the initial collimation, this structure is possibly subject to instabilities which can mass-load and decelerate the outflow, and would also imply a more isotropic - thus less efficient - accelerating field.

iii) Hydromagnetic acceleration: Therefore, hydromagnetic models appear as more promising at least to account for the production of the most powerful jets. Magnetic fields provide a natural mechanical link between disks and jets and can account for the launching, confinement and collimation of jets. The power can be extracted from (and symmetry provided by) the rotation of either/both an accretion disk, giving rise to an MHD wind over a large range in radii [12], [13], or/and - being limited to the inner radii - a spinning black hole threaded by a large scale magnetic field [14]. Much of the current debate involves hydromagnetic models.

Indeed the efficiency of disk vs. black hole energy extraction has been discussed, as the former mechanism may produce more power depending upon the assumptions made [14], [15]. The simplest picture involves the existence of a field component frozen in and threading the disk at large enough angle that matter is centrifugally launched along the field lines. The differential rotation of the disk and inertia of the gas lead to the wrapping up of the field lines, whose hoop stress due to the toroidal component thus generated could then provide the collimation, while the pressure gradient would help the acceleration. Solutions for the structure of the field and resulting MHD flow have been found even for the relativistic case. Although dependent on the inner and outer boundary conditions, they seem to confirm the efficiency of this process in generating collimated flows, asymptotically converting a large fraction of Poynting flux into bulk kinetic power (e.g. [16]), although doubts have been cast on their survival against pinch and helical instabilities [17]. Angular momentum, energy and mass can thus be removed from the accreting flow with an efficiency that depends upon the ratio of the mean value of the open magnetic field to the surface mass density in the disk.

However a good reason to consider still the extraction of the spin energy of the hole is that the high latitude outflow from near a black hole is unlikely to be loaded with baryons, unlike that from an accretion disk corona, and can plausibly attain an ultrarelativistic asymptotic speed. Furthermore as the energy flux is likely to be dominated by the Poynting component close to the hole, radiative drag can be avoided. A particularly attractive picture is that the ultrarelativistic cores of the jets, observed at high radio frequency and $\gamma$-ray energy, are powered by the spin of the hole and collimated by a mildly relativistic hydromagnetic outflow launched by the inner disk, which is, in turn, successively collimated by slower winds from larger radii. Note 
that it is not necessary for the hole power to dominate the disk radiative or hydromagnetic power to account for ultrarelativistic jets, though our current understanding of black hole/disk electrodynamics does not preclude this.

Fundamental questions remain unanswered. They mainly reflect the diffculty in determining the field origin and configuration. The simplest picture involves large scale unipolar fields. Observational evidence for ordered components exists on arcsec and milli arcsec scales (consistent with the effect of shearing), but no indication of the field structure can be inferred for the inner region. It has been suggested that a large field component dragged in from the outer disk might not reach the inner parts as the inflow timescale may exceed the diffusion and reconnection timescales. However, as numerical simulations show [18], following magneto-rotational instabilities in the disk, loops of toroidal and radial field might be generated on a rotational timescale, with scale height comparable to the pressure one. These are then likely to emerge from the disk through buoyancy and reconnect. Interestingly it has been suggested that small scales/unordered field might still provide the requested conditions to launch a jet [19], [20]. The stability of these structures present a further unclear issue. However an interesting possibility is that the formation of a suitable configuration and consequent ejection of matter is non-stationary (e.g. [12], [21]), on the line of what might be hinted from the behavior observed in the micro-quasar GRS $1915+105$.

\section{What We Hope to Learn}

Let us now consider both theoretical and observational issues which currently constitute the most promising steps forward to shed light on the jet formation and the accretion-ejection connection.

- An appealing possibility recently proposed and much debated, is that the formation of jets/outflows might be a natural and necessary condition for accretion to occur. In particular, it has been pointed out that whenever the flow is adiabatic, i.e. radiative dissipation of energy in the flow is inefficient - because either the density is too low or radiation is produced but trapped within the flow due to the long diffusion timescale ([22], [23], [24]) - then energy has to be extracted from the flow mechanically and/or electromagnetically (ADIOS [25]). Hydrodynamic simulations show that the net mass accretion rate increases roughly linearly with radius, though, in the absence of a rapid source of dissipation, the surplus mass escapes as a subsonic breeze rather than a supersonic wind [26]. It will be interesting to see if hydromagnetic simulations exhibit centrifugally-driven super-Alfvénic outflows.

- It is appropriate at this meeting, to remark that the three $\mathrm{X}$-ray observatories, Chandra, XMM and Astro-E, with their complementary capabilities, should revolutionize our understanding of the high energy properties of extragalactic disks and jets. As discussed by [27], $\mathrm{X}$-ray reflection features provide the strongest current evidence for the presence of optically thick disks 
in AGN, thus setting constraints on the geometry and regime of accretion. Iron line profiles are starting to provide a diagnostic of the spacetime around black holes, i.e. a direct measure of their spin, and the system geometry. XMM should allow much improvement and reverberation mapping should become possible with Constellation X or XEUS.

The characteristics of iron lines, strength and profile, are currently of much lower quality in radio-loud objects, for which not even widths can be robustly determined, e.g. [28], [29]. The reflection features appear so far to be comparably weaker than in Seyfert galaxies, and thus still compatible with reprocessing occurring in an optically thin medium (e.g torus, wind). Observations with high sensitivity and spectral resolution broad band X-ray detectors are thus of primary importance.

An independent measurement of the mass and spin of the hole should eventually be provided by QPO in binary X-ray sources, although we do not have a good understanding of how the normal mode frequencies reflect the spacetime geometry, nor of which modes are likely to be excited. Perhaps numerical simulations will be very helpful here. QPO might be starting to be detected in AGN too, on timescales of the order of $\sim 10$ minutes [30].

- Precession of jets has been clearly established only for SS433 and possibly for the blazar OJ 287, e.g. [31], [32]. Other evidence, however, is accumulating from radio imaging of jets which can be interpreted as helical and inverted symmetric structures, possibly originating from precession.

Theoretically the coupling between a disk and a spinning black hole, and in particular the interaction between the two whenever their spin axes are not aligned (Bardeen-Petterson mechanism) is still an open issue. In particular, it has been suggested [33] that the hole might be rapidly spun down by the interaction, thus inhibiting the efficiency of extracting the spin energy of the hole. (Note here the different role that accretion might have in changing the hole mass and spin for galactic and extragalactic objects [34], [35]).

HST imaging indicates that jets and disks are aligned on large scales; higher resolution radio imaging will be necessary to determine what happens closer to the hole and whether the jet is aligned with the spin axis of the disk within the distance at which the hole-disk coupling is plausibly effective.

- As mentioned above, the inner jet speed, as well as changes in velocity field, are crucially linked to the possible baryon loading. Relativistic jet speeds might be even more extreme than commonly assumed.

Recent observations of the jet in M87 have revealed apparent motion on arcsec scales implying $\Gamma \sim 6$, significantly larger than observed before [36].

More extreme bulk velocities have also been invoked to account for extreme values of brightness temperatures inferred from observation of intraday variability at $\mathrm{GHz}$ frequencies, if the emission is due to incoherent synchrotron radiation [37]. $\Gamma>100$ would be needed even in the most conservative case (in terms of total jet power implied [38]) that interstellar refractive scintillation affects the brightness temperature values. Coherent processes would 
also require ad hoc conditions. However new results on variable polarization might imply that more exotic processes are at work [39].

Radio outflows at mildly relativistic velocities are more commonly observed and might be present in as many as $30 \%$ of the radio-quiet sources, although with powers typically three orders of magnitude smaller than in the radio-loud ones. Even more surprising and relevant would be the detection of superluminal motion associated with parsec-scale jets in radio-quiet AGN as recently suggested [40].

- It is clearly important to determine whether jets on different scales can be confined by the external gas or if magnetic fields are needed. Cases of overpressured (powerful) jets have been found, which involve large sections of the flow and thus seem unlikely to be just transient regions. Alternatively, the estimated internal pressure could be lower for a small filling factor of the emitting plasma. Much is expected from the improved (factor $\sim$ ten) spatial resolution of Chandra with regards to the detection of $\mathrm{X}$-ray jets and especially the estimate of the external pressure on small (arcsec) scales, thus possibly determining the scales on which magnetic confinement is indeed compulsory. Note that if magnetic hoop stresses (e.g. in a MHD wind) confine the jet, the required external gas pressure might be reduced by orders of magnitude, corresponding to the radial extension of the wind, i.e. the scale over which the jet is formed and collimated.

Another diagnostic for field confinement would be the determination of its toroidal structure through Faraday rotation mapping, though foreground effects make this quite difficult to carry out in practice.

High resolution radio imaging constitutes also the most direct evidence for the collimation scale. In M87 it has been possible to trace the jet down to scales $\sim 10^{-2}$ pc [41], and recent evidence obtained with high resolution $7 \mathrm{~mm}$ VLBI observations indicates that indeed collimation occurs on these scales, $\sim 60-100 \mathrm{~m}[36]$, thus involving a significant part of the disc.

The degree of collimation could be in principle also determined from statistical arguments within the frame of unification scenarios. However, the likely existence of velocity gradients (polar and possibly radial) limits the robustness of any conclusion.

- A crucial quantity to be determined is obviously the average power and mass flux in jets. Model-dependent estimates can be inferred from the radiative dissipation in the inner jet of blazars and lead to kinetic powers in some cases comparable to and often exceeding the observed radiation from the accreting flow (most conspicuously in low power sources). A further relevant piece of information would be the ratio between the jet power and the Eddington limit: direct mass measures of nearby radio-loud objects might allow this. The emission models adopted assume stationary flows with filling factors of order unity and the results also strongly depending on the low energy end of the particle distribution. Tight constraints on the latter can be derived from the soft X-ray spectrum of high power blazars; here XMM and Astro- 
E should provide the best limits, as well as assess the claimed presence of absorption features by relatively dense and cold gas in and/or around jets.

Closely connected to the power is the jet composition, which is still undetermined. The main initial energy carrier is, possibly, electromagnetic (although this might limit the formation of strong shocks), as this has to be in any case invoked to accelerate and collimate the flow. Radiative constraints from the lack of soft X-ray features exclude a large pair contribution. (Note however that none of the radiative and dynamical constraints on the paucity of pairs seem to apply to low power radio sources). On larger scales, where the bulk of the dissipation occurs and further out on VLBI scales, a significant fraction of the electromagnetic energy is likely to have been converted into kinetic power of an ordinary plasma [42]. Alternatively - as observations of circular polarization might imply [43] - the plasma could be energetically dominated by electron-positron pairs loaded in the jet, although spectral constraints imply that the loading might not be easily achieved [44], [45].

- Galactic superluminal sources provide a promising site to hunt for clues on the disk-jet connection because the variations are so rapid compared with those associated with AGN and it is possible to perform statistical studies with relatively short stretches of data. Furthermore, in these systems a better estimate of the mass inflow might presumably be inferred. Of interest for the galactic vs. extragalactic analogy is the recent determination of highly relativistic bulk velocities in GRS 1915+105 [7], [9].

Indeed GRS $1915+105$ provides the strongest case for a tight inner diskjet connection ([7], [46]), thanks to the detection of episodic accretion-ejection events. These findings strongly call for time dependent models. Nevertheless, jets are also observed in binaries during normal states and transitions between them. Information is still too scarce to infer a clear connection between mass inflow and outflow. In the AGN case the nature of ejection (quasi-stationary or impulsive) is unclear. Flaring events are observed - although with poorly constrained duty cycles - but there is some evidence for a quasi stationary underlying emission component. The corresponding timescales suggest that these events involve only local jet instabilities and no apparent connection with the disk emission has been found. Clues on longer scale trends might be inferred from statistics and the study of young radio sources, e.g. [47].

- Some of the issues concerning the relation between the inflows and outflows will be probably clarified through the interpretation of the results of numerical simulations, which are certainly becoming the necessary support to the understanding of extremely complex physical problems requiring 3D treatment with high dynamical resolution and including MHD, special and general relativity effects. Here great progress has been already achieved on several issues, such as simulation of the behavior of magnetic fields in disks [18], 2D hydrodynamical accreting flows [26], the inner black hole magnetosphere [48] and the propagation of relativistic jets [49]. 
- Finally, let us mention the recent findings [50] concerning the host galaxies of radio-quiet quasars which, contrary to previous belief, appear to be elliptical, as are the hosts of jetted sources. This evidence restricts the parameter space for the origin of the radio-loud/radio-quiet dichotomy, which has now to be ascribed to nuclear or evolutionary properties (e.g. geometry and angular momentum of the inflow, magnetic flux, spin, accretion rate, black hole mass). It is even possible that it is the black hole activity which determines the structure of the host galaxy [51].

One possible speculation is that a rapidly spinning hole is a necessary but not sufficient condition for the formation of a powerful jet, and that a second parameter would be involved, namely the accretion rate over mass ratio [51]. Highly super-Eddington flows could give rise to strong winds (e.g. BAL systems) and strongly ionized disks - accounting for the paucity of Xray reflection features in highly luminous objects [52] - and a strong enough radiation field to inhibit the formation of jets. The latter would instead occur in $\sim$ Eddington limit systems whenever the black hole spins rapidly enough. Very sub-Eddington flows would finally allow for the formation of outflows and jets despite of being radiatively inefficient - as there is growing evidence in low power radio-loud sources.

\section{Acknowledgments}

$A C$ thanks the organizers for setting up this interesting meeting. They are acknowledged together with the MURST for financial support. RB thanks the Institute of Astronomy for hospitality and support through the Beverly and Raymond Sackler Foundation and NASA under grant 5-2837 for support.

\section{References}

1. Begelman M. C., Blandford R. D., Rees M. J. (1984) Rev. Mod. Phys. 56, 255

2. Burgarella D., Livio M., O'Dea C. P. (Eds.) (1993) Astrophysical Jets. Cambridge University Press, Cambridge

3. Ostrowski M., Sikora M. et al. (Eds.) (1997) Relativistic Jets in AGN. Jagellonian University, Cracow

4. Rees M. J. (1971) Nature 229, 312

5. Harris D. E., Biretta J. A., Junor W. (1997) MNRAS 284, L21

6. Gaidos J. A., Akerlof C. W. et al. (1996) Nature 383, 319

7. Fender R. P. (1999) these proceedings

8. King A. R. (1999) these proceedings

9. Mirabel I. F. (1999) these proceedings

10. Blandford R. D., Rees M. J. (1974) MNRAS 169, 395

11. Ghisellini G., Bodo G. et al. (1990) ApJ 362, L1

12. Blandford R. D., Payne D. G. (1982) MNRAS 199, 883

13. Königl A. (1989) ApJ 342, 208

14. Blandford R. D., Znajek R. L. (1977) MNRAS 179, 433

15. Livio M., Ogilvie G. I., Pringle J. E. (1999) ApJ 512, 100

16. Chiueh T., Li Z., Begelman M. C. (1991) ApJ 377, 462 
17. Begelman M. C. (1998) ApJ 493, 291

18. Balbus S. A., Hawley J. F. (1998) Accretion Processes in Astrophysical Systems: Some Like it Hot. Holt S.S., Kallman T.R. (Eds.), AIP Conf Proc. 431, 79

19. Tout C. A., Pringle J. E. (1996) MNRAS 281, 219

20. Heinz S., Begelman M. C. (1999) $19^{\text {th }}$ Texas Symp. on Relativistic Astrophysics and Cosmology. Paul J., Montmerle T., Aubourg E. (Eds.), in press

21. Begelman M. C. (1993) Astrophysical Jets. Burgarella D., Livio M., O'Dea C. P. (Eds.) Cambridge University Press, Cambridge, 305

22. Rees M. J., Begelman M. C., et al. (1982) Nature 295, 17

23. Begelman M. C., Meier D. L. (1982) ApJ 253, 873

24. Narayan R. (1999) this workshop

25. Blandford R. D., Begelman M. C. (1999) MNRAS 303, L1

26. Stone J. M., Pringle J. E., Begelman M. C. (1999) MNRAS, in press

27. Fabian A. C. (1999) this workshop

28. Wozniak P. R., Zdziarski A. A. et al. (1998) MNRAS 299, 449

29. Sambruna R. M., Eracleous M., Mushotzky R. (1999) ApJ, in press

30. Iwasawa K., Fabian A. C. et al. (1998) MNRAS 295, L20

31. Vermeulen R. (1993) Astrophysical Jets. Burgarella D., Livio M., O'Dea C. P. (Eds.), Cambridge University Press, Cambridge, 241

32. Hughes P. A., Aller H. D., Aller M. F. (1999) BL Lac Phenomenon. Takalo L. O., Sillanpää A. (Eds.), ASP Conf. Ser. 159, San Francisco, 273

33. Natarajan P., Pringle J. E. (1998) ApJ 506, L97

34. King A. R., Kolb U. (1999) MNRAS 305, 654

35. Wilson A., Colbert E. J. M. (1995) ApJ 438, 62

36. Junor W., Biretta J. A. (1999) AAS 194.50.17

37. Kedziora-Chudczer L., Jaumcey D. L. et al. (1997) ApJ 490, L9

38. Begelman M. C., Rees M. J., Sikora M. (1994) ApJ 429, L57

39. Macquart J.-P., Kedziora-Chudczer L. et al. (1999) Nature, submitted

40. Blundell K. M., Beasley A. J. (1999) AAS 193.110.04

41. Junor W., Biretta J. A. (1995) AJ 109, 500

42. Celotti A. (1997) Relativistic jets in AGN. Ostrowski M., Sikora M. et al. (Eds.), Jagellonian University, Cracow, 270

43. Wardle J. F. C., Homan D. C. et al. (1998) Nature 395, 457

44. Blandford R. D., Levinson A. (1995) ApJ 441, 79

45. Sikora M., Madejski G. (1999) ApJ, submitted

46. Belloni T., Mendez M., et al. (1997) ApJ 488, L109

47. Reynolds C. S., Begelman M. C. (1997) ApJ 487, L135

48. Koide S., Meier D. L. et al. (1999) ApJ, submitted

49. Aloy M. A., Ibanez J. M. A. et al. (1999) ApJ 523, L125

50. McLure R. J., Kukula M. J. et al. (1999) MNRAS 308, 377

51. Blandford R. D. (1999) Royal Society, London

52. Fabian A. C. (1998) Accretion Processes in Astrophysical Systems: Some Like it Hot. Holt S.S., Kallman T.R. (Eds.) AIP Conf Proc. 431, 246 